\title{
Effect of traditional Chinese medicine on serum inflammation and efficacy in patients with sepsis: a systematic review and meta- analysis
}

\author{
Yituo Wen ${ }^{1 \#}$, Changfu Feng ${ }^{2 \#}$, Wei Chen ${ }^{3}$, Changneng Chen ${ }^{2}$, Shunling Kuang ${ }^{2}$, Fang Liu ${ }^{1}$, \\ Qiufeng Tang ${ }^{1}$, Miancong Chen ${ }^{3}$
}

${ }^{1}$ Department of Pharmacy, Cadre Sanatorium of Hainan \& Geriatric Hospital of Hainan (CSH), Haikou, China; ${ }^{2}$ Department of Second District of Critical Medicine, The Second Affiliated Hospital of Hainan Medical University, Haikou, China; ${ }^{3}$ Department of Critical Medicine, The First Affiliated Hospital of Hainan Medical University, Haikou, China

Contributions: (I) Conception and design: Y Wen; (II) Administrative support: M Chen; (III) Provision of study materials or patients: C Feng; (IV) Collection and assembly of data: W Chen; (V) Data analysis and interpretation: Y Wen; (VI) Manuscript writing: All authors; (VII) Final approval of manuscript: All authors.

"These authors contributed equally to this work.

Correspondence to: Miancong Chen. Department of Critical Medicine, The First Affiliated Hospital of Hainan Medical University, 31 Longhua Road, Longhua District, Haikou 570102, China. Email: chenmiancong998@163.com.

Background Sepsis is a systemic infectious inflammatory response syndrome, which can release a large number of inflammatory mediators in serum, resulting in organ dysfunction. This study conducted a systematic review and meta-analysis of the reports of traditional Chinese medicine (TCM) in the treatment of sepsis in recent years, providing evidence for clinical diagnosis and treatment.

Methods: The databases of PubMed, Chinese Medical Literature (CBM), China National Knowledge Infrastructure (CNKI), Medline, and Web of Science were searched for clinical randomized controlled trials (RCTs) related to "Chinese traditional medicine" and "Sepsis" published from January 2015 to August 2021. After screening qualified articles, RevMan 5.4 software was used to evaluate the bias of included studies and perform meta-analysis.

Results: Finally, 8 articles were retrieved and screened, and a total of 862 patients entered the study. The results of meta-analysis showed that serum interleukin-6 (IL-6) levels were lower in sepsis patients after TCM treatment [mean difference $(M D)=-20.70 ; 95 \%$ confidence interval $(\mathrm{CI}):-33.68$ to $-7.73 ; \mathrm{P}=0.002$ ]; tumor necrosis factor- $\alpha(\mathrm{TNF}-\alpha)$ levels were lower $(\mathrm{MD}=-4.17 ; 95 \% \mathrm{CI}:-5.98$ to $-2.37 ; \mathrm{P}<0.00001)$; acute physiological assessment and chronic health evaluation (APACHE) II scores were lower (MD =-2.92; 95\% CI: -3.50 to $-2.35 ; \mathrm{P}<0.00001)$; mortality was lower [odds ratio (OR) $=0.49 ; 95 \% \mathrm{CI}$ : 0.33 to 0.74 ; $\mathrm{P}=0.0006]$; and effective rate was higher ( $\mathrm{OR}=3.43 ; 95 \% \mathrm{CI}: 1.72$ to $6.85 ; \mathrm{P}=0.0005)$.

Discussion: On the basis of traditional western medicine treatment of sepsis, the addition of TCM treatment can effectively reduce the serum inflammatory response, improve the patient's immunity, and improve treatment efficacy.

Keywords: Traditional Chinese medicine (TCM); sepsis; inflammation; efficacy; meta-analysis

Submitted Oct 18, 2021. Accepted for publication Nov 16, 2021.

doi: 10.21037/apm-21-3179

View this article at: https://dx.doi.org/10.21037/apm-21-3179 


\section{Introduction}

Sepsis is a systemic infectious inflammatory response syndrome that can lead to tissue hypoperfusion, organ dysfunction, and even irreversible persistent hypotension, known as septic shock, with a mortality rate as high as $80 \%$ (1). Sepsis is frequently seen in the intensive care unit (ICU) and is a common complication of severe burn, trauma, severe infection, and surgery. The body is invaded by endotoxins and pathogens, releasing a large number of inflammatory mediators, increasing the expression of serum inflammatory factors [interleukin-2 (IL-2), IL-6, tumor necrosis factor- $\alpha$ (TNF- $\alpha$ ), C-reactive protein (CRP)], disturbing the immune system and antiinflammatory system, dysregulating the intestinal flora, causing coagulation disorders, and damaging organ function (2). The decline of autoimmunity, the use of cytotoxic drugs, surgery, invasive examination and chronic diseases are common causes of sepsis, the treatment of the disease is mainly based on the control of infection and mechanical ventilation adjuvant therapy, but conventional treatment cannot rapidly control the serum inflammatory response and regulate the body's immune capacity. Traditional Chinese medicine (TCM) has accumulated a wealth of experience in the treatment of sepsis. The constituents of traditional Chinese herbal medicine have the properties of clearing away heat and toxic substances, destroying bacteria, regulating immunity, and promoting the reconstruction of neuroendocrine network system (3). In their study, Lin et al. (4), applied the traditional Chinese herbal formula Bai-hu-tang via injection to rats with sepsis, and it was found that the serum levels of IL-6 and IL-10 in rats after injection were significantly lower, while the survival rate was improved. In the meta-analysis by Liang et al. (5), 10 randomized controlled trials (RCTs) were included for analysis, and the conclusion was that the addition of TCM in the treatment of sepsis could yield a better therapeutic effect. However, the quality of RCTs included in their meta-analysis was not high, the risk of bias was great, and the included studies were published a long time ago. In the current study, 8 high-quality articles published from 2015 to 2021 were retrieved and included to further explore this topic. We present the following article in accordance with the PRISMA reporting checklist (available at https://dx.doi.org/10.21037/apm-21-3179).

\section{Methods}

\section{Literature search}

(I) Search strategy: the subject search was defined by the search terms "TCM" or "traditional Chinese medicine" or "Chinese" AND "Sepsis" or "Septic Shock" or "Sepsis Induced"; (II) database: the databases of PubMed, Chinese Medical Literature (CBM), China National Knowledge Infrastructure (CNKI), Medline, and Web of Science were searched. We believed that these five databases cover most of the studies on the impact of TCM on sepsis; (III) filter setting was conducted as follows: set the time of database, filter the literature type, filter out RCTs, and limit the publication time between January 2015 and August 2021.

\section{Literature inclusion criteria}

Inclusion criteria were conducted with reference to the population, intervention, control, and outcomes (PICO) principle of evidence-based medicine (i.e., P: participant; I: intervention; C: control; O: outcome). Setting inclusion criteria: (I) participants: all patients were aged over 18 years (excluding animal studies). All patients were diagnosed with sepsis, severe sepsis or septic shock, meeting the diagnostic criteria defined in the Third International Consensus Definition of Sepsis and Septic Shock proposed in 2016 (6); (II) intervention measures: all studies were divided into control group and experimental group for intervention. Both groups were given the same conventional treatment (hemodynamic monitoring, infection control, fluid resuscitation, mechanical ventilation, nutritional support), the only difference between the two groups of patients was the addition of TCM treatment in the experimental group, including: TCM decoction, acupuncture, bundle therapy; (III) control methods: the literature study type was RCT, articles with complete preferred randomization method, allocation concealment, and blind method; (IV) outcome indicators: complete statistical method, clear data could be obtained, including outcome indicators: (i) inflammatory indicators, including serum IL-6, TNF- $\alpha$, CRP levels after treatment; (ii) immune indicators: including $\mathrm{CD}^{+}$and $\mathrm{CD}^{+}$after treatment; (iii) efficacy indicators, including duration of ICU stay, mortality, effective rate, acute 
physiological assessment and chronic health evaluation (APACHE) II score; and (iv) adverse reactions; (V) observation time: the observation points were set at 1, 3, and 7 days after treatment for serological indicators. The data in this analysis were collected from the data at 7 days after treatment. The efficacy and mortality were limited to 28 days after admission.

\section{Literature exclusion criteria}

(I) Intervention group participants were human; had comorbid coagulation disorders, tumors, blood diseases, or immune system diseases; allergic to TCM; pregnant women or organ transplant patients; (II) outcome indicators were not clearly described, or data was not available; (III) nonRCT.

\section{Selection of studies}

After two researchers had independently completed the literature retrieval, the data were entered into NoteExpress for subsequent management, and duplicate articles were filtered out. Then, titles, abstracts, and full texts were read, and unqualified articles were excluded. If there was any dispute between researchers in this process, a 3rd person was called to intervene and coordinate after discussion.

\section{Literature risk of bias assessment}

Using the risk of bias assessment module built in RevMan 5.4 software (RevMan; the Cochrane Collaboration, Copenhagen, Denmark, 2020) to assess the following indicators: (I) random allocation method; (II) blind method; (III) implementation of allocation concealment; (IV) data integrity; (V) selective reporting bias; (VI) other biases, including "low risk", "unclear", and "high risk".

\section{Data extraction and conversion}

After completing the literature selection and bias assessment, two researchers read the full texts again, extracting the following information: article (author, publication time), participants (number of infected organs, source of infection, complications, gender ratio, age), intervention measures (number of patients in groups, intervention methods), and outcomes (outcome indicators).

\section{Statistical methods}

(I) RevMan 5.4 was used for meta-analysis; (II) standard mean difference (SMD), effect size, and 95\% confidence interval (CI) were used for continuous indicators, odds ratio (OR), effect size, and $95 \% \mathrm{CI}$ were used for discrete indicators (i.e., binary classification), and $\mathrm{P}<0.05$ indicated statistical significance; (III) each outcome indicator was analyzed; (IV) forest plot was used to present the effect size; (V) $\mathrm{I}^{2}$ analysis and $\mathrm{Q}$ were used to verify literature heterogeneity, $\mathrm{I}^{2}>50 \%$ or $\mathrm{P}<0.1$ indicated heterogeneity and the random effects model was used; otherwise, the fixed effects model was used, Mantel-Haenszel model was used for OR effect size, and inverse model was used for SMD effect size; (VI) descriptive analysis was only performed when heterogeneity between studies was suggested and the source of heterogeneity could not be judged; (VII) sensitivity analysis was performed for results; (VIII) funnel plot was used to represent publication bias.

\section{Results}

\section{Literature screening process and results}

The flow chart of file retrieval is shown in Figure 1. After the initial retrieval of 361 articles and subsequent screening, 8 articles were selected and included, and a total of 862 patients were included in the study.

\section{Basic characteristics of literatures}

The basic characteristics and interventions, treatment time, and outcome indicators of the included articles are shown in Table 1, with 6 Chinese herbal decoctions, 1 acupuncture, and 1 bundle treatment used for the intervention methods. In Table 2, statistics of included articles for main indicators are shown.

\section{Literature bias assessment}

In this study, all articles indicated that the randomization method was used, but the grouping bias caused by the randomization method was small; some articles $(8,11,13)$ did not indicate whether the allocation concealment and blind method were used, which may have increased the risk of implementation bias; some articles $(8,11,13)$ did not record the data of dropout cases in detail, which may have 


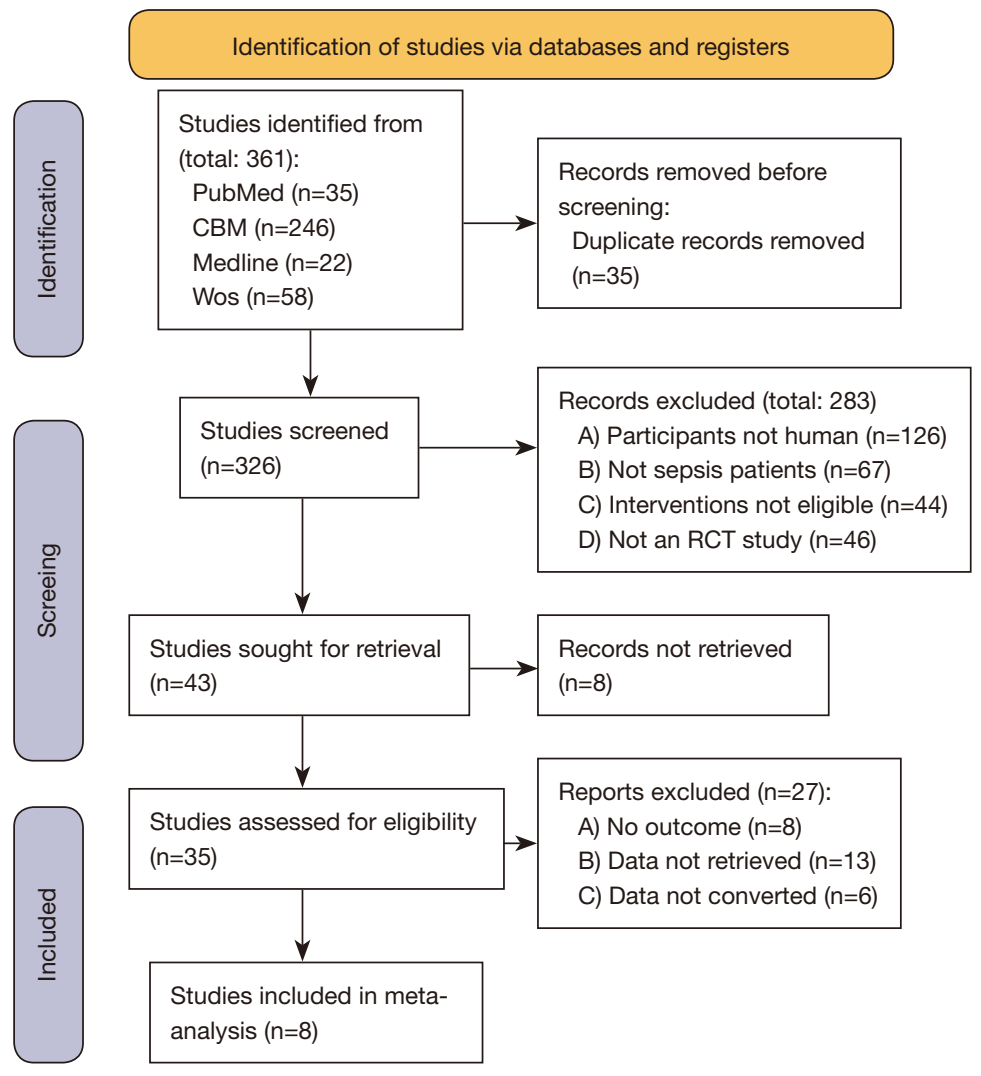

Figure 1 Literature selection flow chart. CBM, Chinese Medical Literature; CNKI, China National Knowledge Infrastructure; RCT, randomized controlled trial.

cause incompleted data; there was no selective reporting or other bias (Figures 2,3).

\section{Meta-analysis results}

\section{IL-6}

A total of 4 articles reported the effect of TCM on IL-6 in sepsis patients, with a total of 122 experimental group participants and 121 control group participants, and there was statistical heterogeneity between the 4 articles $\left(\mathrm{I}^{2}=98 \% ; \mathrm{P}<0.00001\right)$. Using random-effects model analysis, the pooled effect size was mean difference $(\mathrm{MD})=-20.70,95 \% \mathrm{CI}:-33.68$ to -7.73 , indicating that serum IL-6 level in sepsis patients was lower after TCM treatment, which was statistically significant $(\mathrm{Z}=3.13$; $\mathrm{P}=0.002)$, as shown in Figure 4.

\section{TNF- $\alpha$}

A total of 5 articles reported the effect of TCM on TNF- $\alpha$ in sepsis patients, 273 participants were included in the experimental group and 263 participants in the control group, and 5 articles were statistically heterogeneous $\left(\mathrm{I}^{2}=97 \% ; \mathrm{P}<0.00001\right)$. Using random-effects model analysis, the pooled effect size was MD $=-4.17,95 \% \mathrm{CI}$ : -5.98 to -2.37 , which was statistically significant $(Z=4.53$; $\mathrm{P}<0.00001)$, indicating that TNF- $\alpha$ levels were lower in sepsis patients after TCM treatment, as shown in Figure 5.

\section{APACHE II score}

A total of 7 articles reported the effect of TCM on APACHE II score in sepsis patients, including 280 cases in the experimental group and 280 cases in the control group, and no statistical heterogeneity was detected among these studies $\left(\mathrm{I}^{2}=40 \% ; \mathrm{P}=0.12\right)$. Using fixed-effects model analysis, the pooled effect size was $M D=-2.92,95 \%$ CI: -3.50 to -2.35 , with statistical significance $(Z=9.97$; $\mathrm{P}<0.00001$ ), indicating that APACHE II score was lower in sepsis patients after TCM treatment.

We further divided the study into four subgroups 


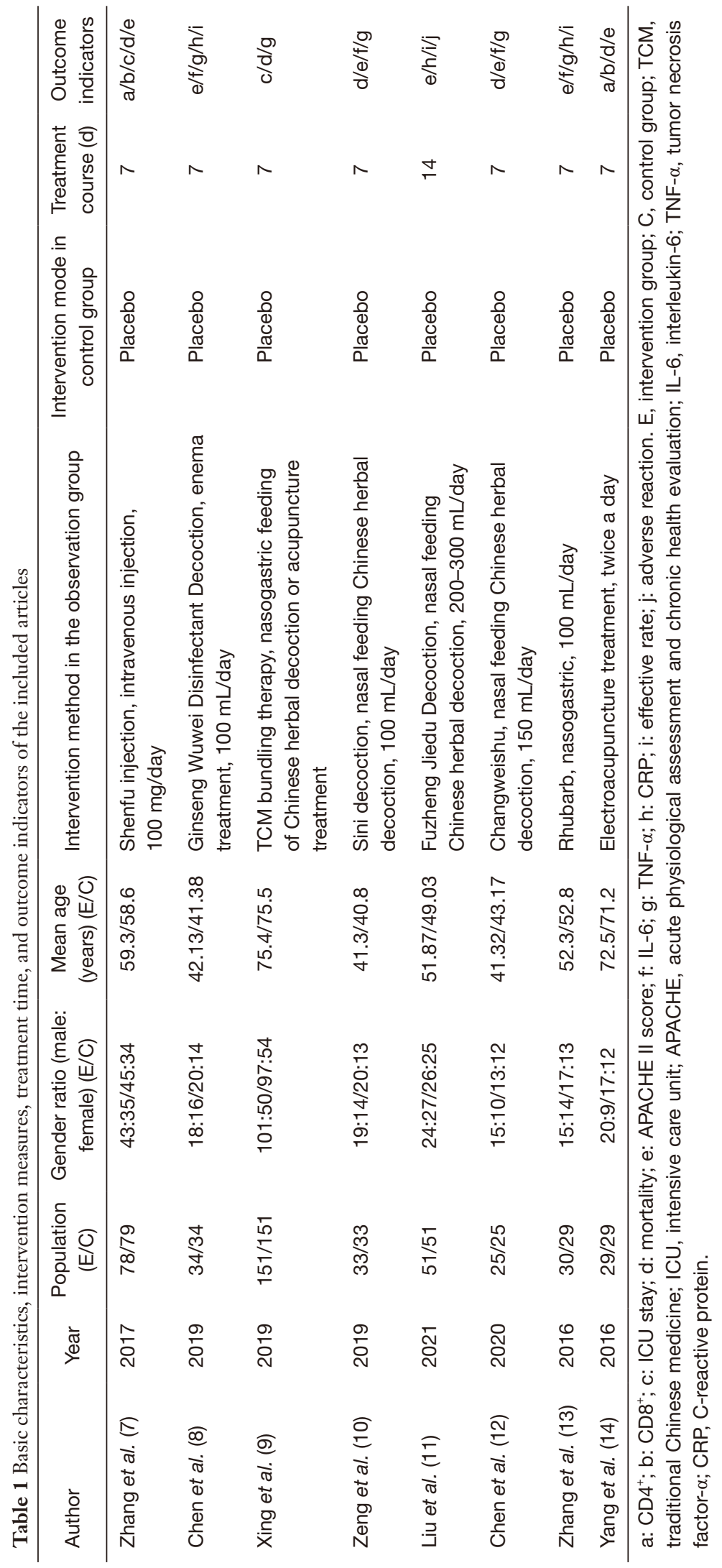


Table 2 Statistics of included articles for main indicators

\begin{tabular}{lccc}
\hline Metrics & Statistical unit & Number of reported literatures & Reported literatures \\
\hline CD4 $^{+}$ & $\mathrm{pg} / \mathrm{mL}$ & 2 & $(7,14)$ \\
$\mathrm{CD}^{+}$ & $\mathrm{pg} / \mathrm{mL}$ & 2 & $(7,14)$ \\
$\mathrm{IL}-6$ & $\mathrm{ng} / \mathrm{L}$ & 4 & $(8,10,12,13)$ \\
TNF- $\alpha$ & $\mathrm{Mg} / \mathrm{mL}$ & 5 & $(8-10,12,13)$ \\
CRP & $\mathrm{mg} / \mathrm{L}$ & 3 & $(8,11,13)$ \\
APACHE II score & - & 7 & $(7,8,10-14)$ \\
Mortality & $\%$ & 5 & $(7,9,10,12,14)$ \\
ICU stay & $\mathrm{d}$ & 2 & $(7,9)$ \\
Effective rate & $\%$ & 3 & $(8,11,13)$ \\
\hline
\end{tabular}

IL-6, interleukin-6; TNF- $\alpha$, tumor necrosis factor- $\alpha$; CRP, C-reactive protein; APACHE, acute physiological assessment and chronic health evaluation; ICU, intensive care unit.
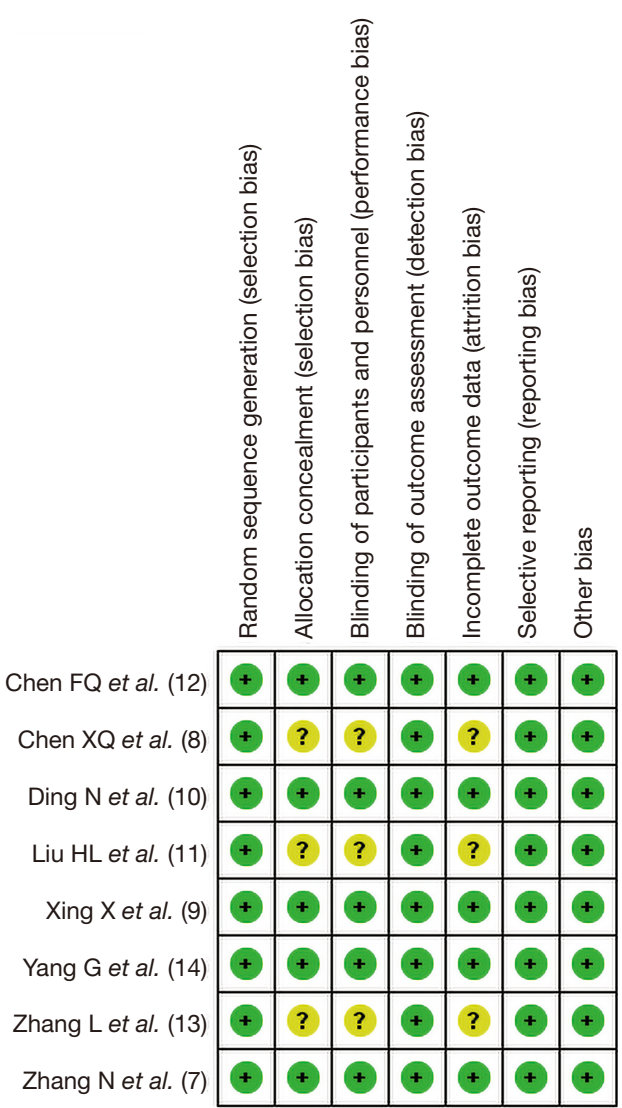

Figure 2 Risk of bias summary (7-14). according to TCM intervention methods: intravenous injection, TCM decoction, enema treatment and acupuncture. The four intervention methods can significantly improve the APACHE II score of patients after treatment, and the MD effect is statistically significant, as shown in Figure 6.

Due to the statistical heterogeneity within the subgroup of TCM decoction $\left(\mathrm{I}^{2}=67 \% ; \mathrm{P}=0.03\right)$, we excluded the literature one by one for further investigation. After removing the literature (13), the internal heterogeneity of the remaining three studies (10-12) disappeared $\left(\mathrm{I}^{2}=0 \%\right.$; $\mathrm{P}=0.67)$. It can be seen that the literature (13) is the source of internal heterogeneity.

\section{Mortality}

A total of 5 articles reported the effect of TCM on the mortality of sepsis patients, including 316 patients in the experimental group and 317 patients in the control group, and no statistical heterogeneity was detected among the 5 articles $\left(\mathrm{I}^{2}=0 \% ; \mathrm{P}=0.68\right)$. The fixed-effect model was used for analysis, and the pooled effect size was OR $=0.49,95 \%$ CI: 0.33 to 0.74 , with statistical significance $(\mathrm{Z}=3.44 ; \mathrm{P}=0.0006)$, indicating that the mortality rate of sepsis patients was lower in TCM treatment, as shown in Figure 7. 


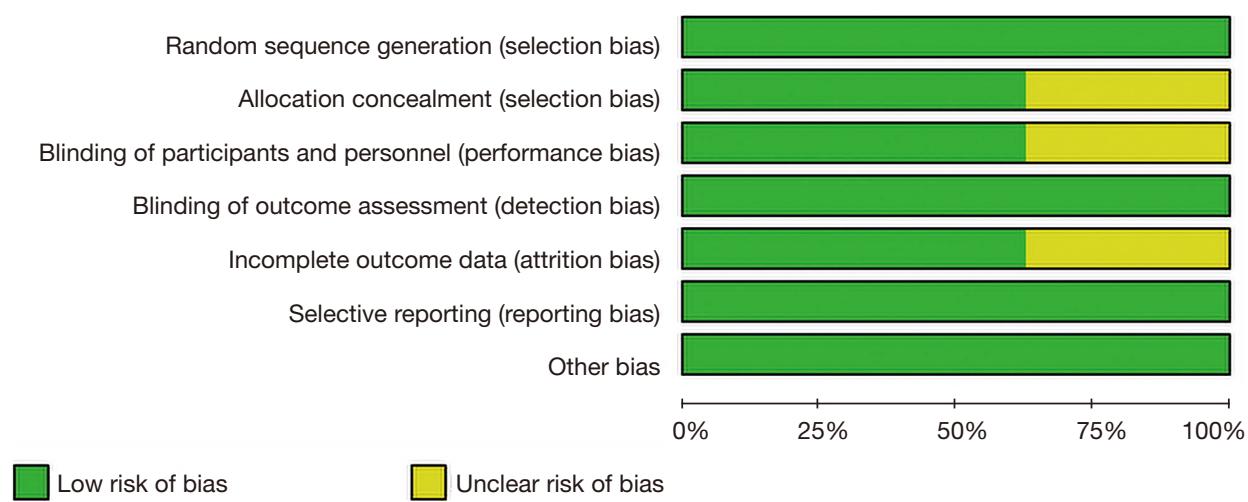

Figure 3 Risk of bias graph.

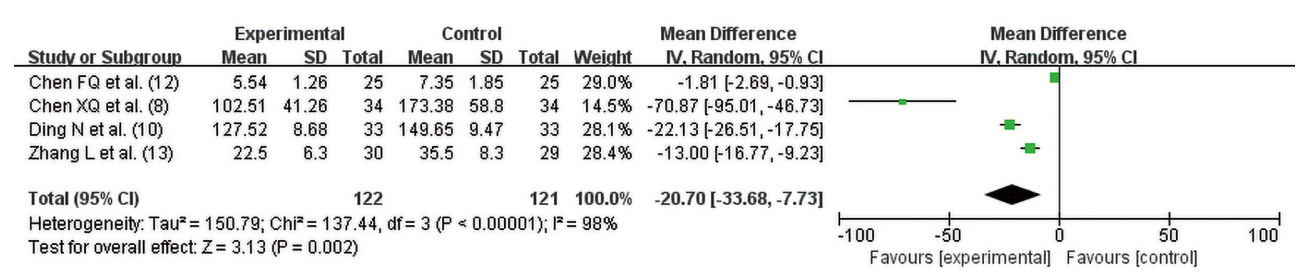

Figure 4 Combined analysis of the effect of TCM on IL-6 in patients with sepsis $(8,10,12,13)$. TCM, traditional Chinese medicine; IL-6, interleukin-6; SD, standard deviation; CI, confidence interval.

\begin{tabular}{|c|c|c|c|c|c|c|c|c|c|c|c|c|}
\hline \multirow[b]{2}{*}{ Studv or Subaroup } & \multicolumn{3}{|c|}{ Experimental } & \multicolumn{3}{|c|}{ Control } & & \multirow{2}{*}{$\begin{array}{l}\text { Mean Difference } \\
\text { N, Random, } 95 \% \mathrm{Cl}\end{array}$} & \multirow{2}{*}{\multicolumn{4}{|c|}{$\begin{array}{c}\text { Mean Difference } \\
\text { IV. Random, } 95 \% \mathrm{Cl}\end{array}$}} \\
\hline & Mean & SD & Total & Mean & SD & Total & Weight & & & & & \\
\hline Chen FQ et al. (12) & 5.38 & 1.19 & 25 & 10.89 & 1.65 & 25 & $26.5 \%$ & $-5.51[-6.31,-4.71]$ & $\square$ & & & \\
\hline Chen XQ et al. (8) & 0.41 & 0.06 & 34 & 1.58 & 0.55 & 34 & $27.9 \%$ & $-1.17[-1.36,-0.98]$ & & & & \\
\hline Ding $N$ et al. $(10)$ & 7.83 & 0.81 & 33 & 9.16 & 0.95 & 33 & $27.5 \%$ & $-1.33[-1.76,-0.90]$ & & & & \\
\hline Xing $\times$ et al. (9) & 112.3 & 31.8 & 151 & 122.7 & 36.5 & 151 & $4.6 \%$ & $-10.40[-18.12,-2.68]$ & - & & & \\
\hline Zhang L et al. (13) & 8.2 & 4.6 & 30 & 19.6 & 7.1 & 20 & $13.5 \%$ & $-11,40[-14.92,-7.88]$ & - & & & \\
\hline Total (95\% Cl) & & & 273 & & & 263 & $100.0 \%$ & $-4.17[-5.98,-2.37]$ & 1 & & & \\
\hline $\begin{array}{l}\text { Heterogeneity: Tauz } \\
\text { Test for overall effec }\end{array}$ & $\begin{array}{l}3.04 ; \mathrm{Cr} \\
Z=4.53\end{array}$ & $\begin{array}{l}h i^{2}=14 \\
(P \leq 0\end{array}$ & $\begin{array}{l}44.22, d \\
1.00001)\end{array}$ & $d f=4(P$ & $\Leftrightarrow 0.00$ & $0001) ; P^{2}$ & $=97 \%$ & & $\begin{array}{ll}1 \\
-100 & -50 \\
& \text { Favours [experimental] }\end{array}$ & Favours & $\begin{array}{l}50 \\
\text { ontroll] }\end{array}$ & 100 \\
\hline
\end{tabular}

Figure 5 Combined analysis of the effect of TCM on TNF- $\alpha$ in patients with sepsis $(8-10,12,13)$. TCM, traditional Chinese medicine; TNF- $\alpha$, tumor necrosis factor- $\alpha$; SD, standard deviation; CI, confidence interval.

\section{Other indicators}

Effects of other indicators such as $\mathrm{CD}^{+}$and $\mathrm{CD} 8^{+}$are shown in Table 3.

\section{Heterogeneity investigation and sensitivity analysis}

For IL-6/TNF- $\alpha$ with greater heterogeneity in the study, it was found that the main source of heterogeneity was that the units used for IL-6/TNF- $\alpha$ statistics were different between the studies, so the data were different. For example, an article (8) used $\mathrm{ng} / \mathrm{L}$ as the unit, while another (10) used $\mathrm{pg} / \mathrm{mL}$ as the unit. We then analyzed APACHE II score, as it had less heterogeneity, by random effects model and fixed effects model, respectively. The result was similar, which supported that the analysis was stable.

In addition, according to the intervention methods, the seven studies included for APACHE II score were divided into four subgroups: intravenous injection (including 1 study), TCM decoction (including 4 studies), enema treatment (including 1 study) and acupuncture (including 1 study). Among them, there was statistical heterogeneity in the decoction subgroup. After excluding Literature (13), the heterogeneity of the remaining three studies disappeared, which meant that the heterogeneity was 


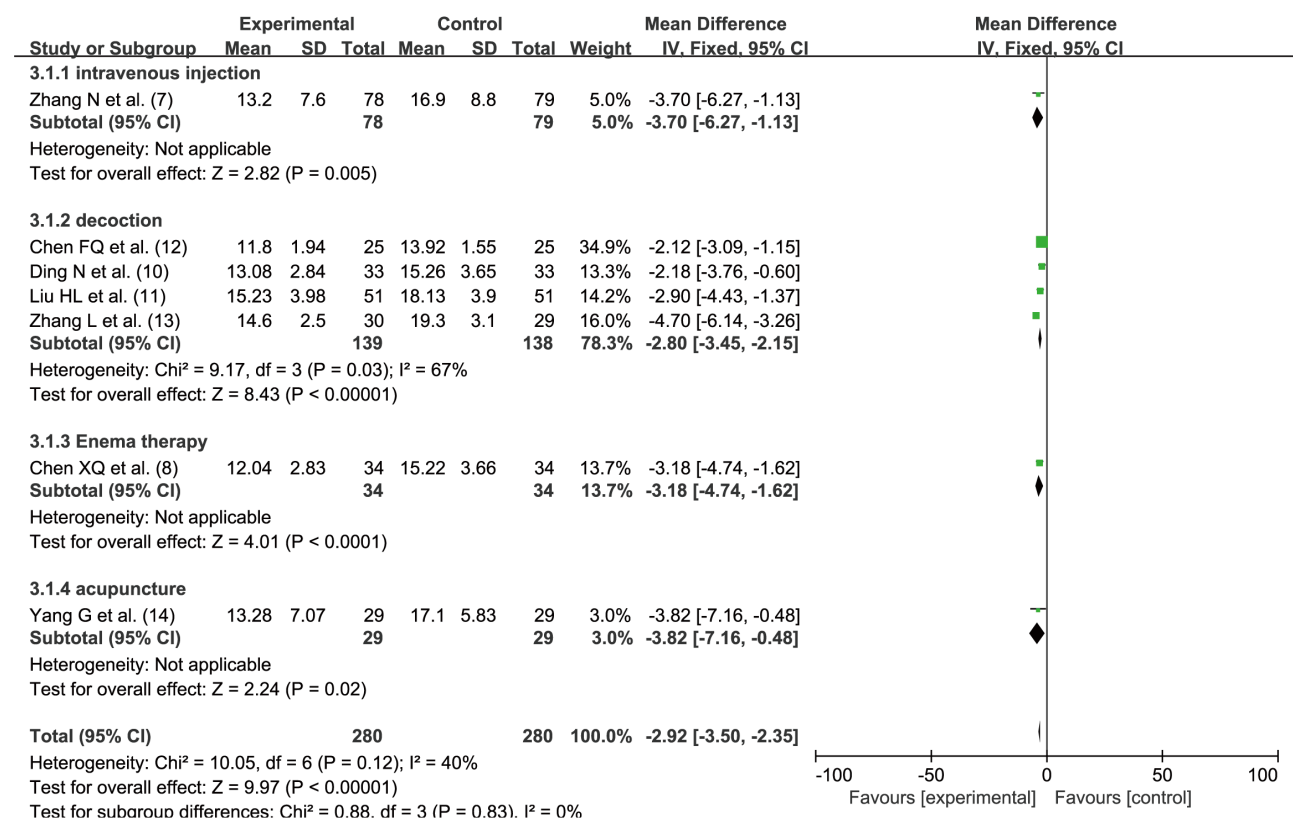

Figure 6 Combined analysis of the effect of TCM on APACHE II score in patients with sepsis $(7,8,10-14)$. TCM, traditional Chinese medicine; APACHE, acute physiological assessment and chronic health evaluation; SD, standard deviation; CI, confidence interval.

\begin{tabular}{|c|c|c|c|c|c|c|c|c|c|}
\hline Studv or Subgroup & $\begin{array}{l}\text { Experim } \\
\text { Events }\end{array}$ & $\begin{array}{l}\text { ental } \\
\text { Total }\end{array}$ & $\begin{array}{l}\text { Contro } \\
\text { Events }\end{array}$ & ol & Weight & $\begin{array}{c}\text { Odds Ratio } \\
\text { M-H. Fixed, 95\% Cl }\end{array}$ & $\begin{array}{r}\text { Odds F } \\
\text { M-H. Fixed }\end{array}$ & $\begin{array}{l}\text { Ratio } \\
\text { d. } 95 \% \mathrm{Cl}\end{array}$ & \\
\hline Chen FQ et al. (12) & 3 & 25 & 8 & 25 & $10.3 \%$ & $0.29[0.07,1.26]$ & \begin{tabular}{l|l} 
&
\end{tabular} & - & \\
\hline Ding $N$ et al. (10) & 0 & 33 & 4 & 33 & $6.5 \%$ & $0.10[0.01,1.89]$ & & & \\
\hline Xing $X$ et al. (9) & 21 & 151 & 37 & 151 & $46.7 \%$ & $0.50[0.28,0.90]$ & & & \\
\hline Yang $G$ et al. (14) & 5 & 29 & 9 & 29 & $10.9 \%$ & $0.46[0.13,1.61]$ & & 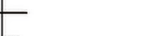 & \\
\hline Zhang $N$ et al. $(7)$ & 16 & 78 & 22 & 79 & $25.5 \%$ & $0.67[0.32,1.40]$ & & & \\
\hline Total $(95 \% \mathrm{Cl})$ & & 316 & & 317 & $100.0 \%$ & $0.49[0.33,0.74]$ & & & \\
\hline Total events & 45 & & 80 & & & & & & \\
\hline \multicolumn{7}{|c|}{$\begin{array}{l}\text { Heterogeneity: } \mathrm{Chi}^{2}=2.32, d f=4(P=0.68) ; l^{2}=0 \% \\
\text { Test for overall effect: } Z=3.44(P=0.0006)\end{array}$} & $\begin{array}{lll}0.01 & 0.1 \\
& \text { Favours [experimental] }\end{array}$ & $\begin{array}{cc}10 \\
\text { Favours [control] }\end{array}$ & 100 \\
\hline
\end{tabular}

Figure 7 Combined analysis of the effect of TCM on the mortality of patients with sepsis $(7,9,10,12,14)$. TCM, traditional Chinese medicine; CI, confidence interval.

caused by literature (13), It may be related to its single formula (rhubarb), which was different to other decoction with combined formula.

Since the remaining three studies (10-12) are homogeneous and the dosage is all within $100-300 \mathrm{~mL} / \mathrm{d}$, we did not conduct further subgroup analysis according to the dosage.

\section{Publication bias analysis}

Since the largest number of articles $(n=7)$ was included in the analysis of APACHE II score, the funnel plot for this analysis was calculated to show that the articles were evenly distributed on both sides, suggesting that the possibility of publication bias was small, as shown in Figure 8.

\section{Discussion}

Sepsis is a common clinical critical disease, but its pathogenesis is still not sufficiently clear. Modern medicine believes that its predisposing causes may be related to the interaction of inflammatory mediators, colonization and translocation of intestinal bacteria, gene polymorphisms, and other factors (15). The main manifestation of sepsis is a systemic uncontrolled inflammatory response in which a large number of cytokines and inflammatory mediators are released into the serum, causing cellular and tissue trauma, 
Table 3 Meta-analysis results of other indicators

\begin{tabular}{|c|c|c|c|c|c|c|}
\hline Factors & $\begin{array}{c}\text { Number of } \\
\text { participating patients }\end{array}$ & $\begin{array}{l}\text { Number of } \\
\text { articles }\end{array}$ & Statistical method & $\mathrm{I}^{2}$ with $\mathrm{P}$ value & Effect estimate & $P$ value \\
\hline $\mathrm{CD}^{+}$ & 215 & 2 & MD (IV, fixed, 95\% Cl) & $0 \%$ with 0.43 & $9.98(7.25,12.71)$ & $<0.00001$ \\
\hline $\mathrm{CD}^{+}$ & 215 & 2 & MD (IV, fixed, 95\% Cl) & $0 \%$ with 0.96 & $87.32(66.62,108.03)$ & $<0.00001$ \\
\hline Effective rate & 229 & 3 & OR (M-H, fixed, 95\% Cl) & $0 \%$ with 0.85 & $3.43(1.72,6.85)$ & 0.0005 \\
\hline ICU stay time & 459 & 2 & MD (IV, fixed, 95\% Cl) & $0 \%$ with 0.37 & $-1.84(-2.73,-0.96)$ & $<0.0001$ \\
\hline
\end{tabular}

CRP, C-reactive protein; ICU, intensive care unit; MD, mean difference; Cl, confidence interval; OR, odds ratio.

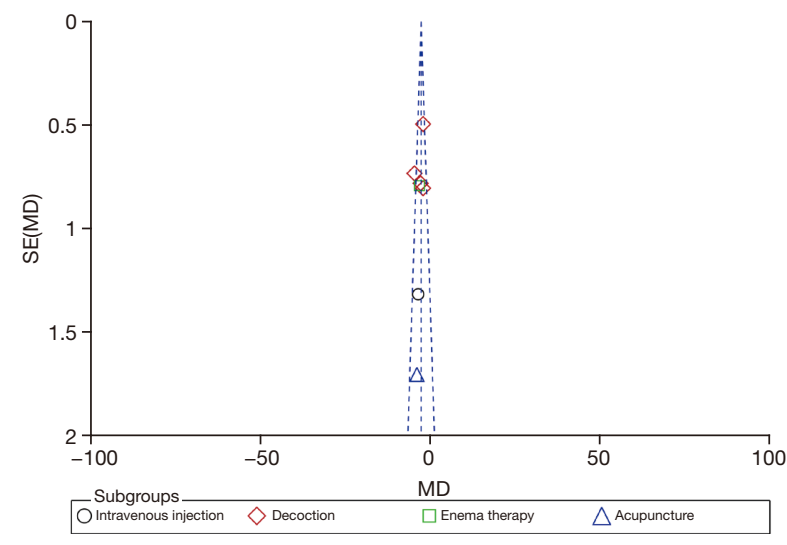

Figure 8 Funnel plot. SE, standard error; MD, mean difference.

and resulting in organ dysfunction (16). Serum IL-6, TNF- $\alpha$, and CRP are the most common inflammatory factors in this response, and during the acute phase of infection, serum levels of IL-6, TNF- $\alpha$, and CRP are markedly increased and waterfall effects can be induced, whereas when infection is controlled, they are markedly decreased (17). In this metaanalysis, 8 articles using TCM treatment (including TCM decoction, acupuncture, and TCM bundle treatment) were included, and the results showed that the serum levels of IL-6 and TNF- $\alpha$ in sepsis patients after TCM treatment were significantly lower than those in the control group (placebo). The measurement time points were selected from 7 days after treatment, which suggested that in the conventional western medical treatment, the addition of TCM treatment could accelerate the control of inflammatory response in a short time, thereby improving the condition. The addition of TCM did not play a role in reducing CRP in this study, which may have been related to the paucity of included articles (only 2). The APACHE II scoring system enables a comprehensive assessment of the condition of sepsis patients and can reflect the condition for clinical reference (18). In this meta-analysis, a total of 7 articles reported the effect of the addition of TCM on improving participant APACHE II scores, and 5 articles reported the effect of the addition of TCM on reducing patient mortality. Although only 2 articles reporting $\mathrm{CD}^{+}{ }^{+}$and $\mathrm{CD} 8^{+}$were included, the results showed that the addition of TCM had a promoting effect on improving patient immunity and could shorten the length ICU stay.

In this study, a total of 6 articles used TCM decoction (whereby TCM was made into decoction, nasogastric feeding therapy, or enema therapy). The main components in the decoction included ginseng, honeysuckle, rhubarb, dandelion, wild chrysanthemum, and so on. The existing pharmacology confirmed that ginseng has the effect of regulating immune function and the neuroendocrine system (19); boneysuckle is a good medicine for clearing away heat and toxic substances, which can remove endotoxin and improve the body's immunity (8); wild chrysanthemum and dandelion can sterilize, having the effect of detoxification and diuresis (11); rhubarb helps to repair intestinal microecology, inhibit bacterial reproduction, and improve gastrointestinal function, thereby reducing inflammation (13). A study (14) used acupuncture to stimulate specific acupoints, improving the immunity of sepsis patients and improving clinical treatment outcomes. In another study (9), TCM bundle therapy was used, whereby according to the theory of TCM syndrome differentiation and treatment and the different symptoms of sepsis patients, different prescriptions of TCM decoction gavage or TCM acupuncture treatment were administered, to target and amplify the effect of TCM (20).

In this study, some of the 8 included studies did not describe the allocation concealment, blind method, or count the drop-out cases, and so on, which may have resulted in 
some bias; however, the overall quality was good. In the analysis of IL-6/TNF- $\alpha$ and other indicators, there was a large heterogeneity between the articles, which may have been caused by the different units used in the statistical data of each study. Part of the indicator (CRP) had insufficient evidence due to too few included articles, and in future studies, more studies with higher quality need to be included to provide stronger evidence.

\section{Conclusions}

In the traditional western medicine treatment of sepsis, the addition of TCM treatment can effectively reduce the serum inflammatory response of patients, improve the patient's immunity, and improve the efficacy. However, more high-quality RCTs are needed in clinical practice to provide stronger evidence.

\section{Acknowledgments}

Funding: None.

\section{Footnote}

Reporting Checklist: The authors have completed the PRISMA reporting checklist. Available at https://dx.doi. org/10.21037/apm-21-3179

Conflicts of Interest: All authors have completed the ICMJE uniform disclosure form (available at https://dx.doi. org/10.21037/apm-21-3179). The authors have no conflicts of interest to declare.

Ethical Statement: The authors are accountable for all aspects of the work in ensuring that questions related to the accuracy or integrity of any part of the work are appropriately investigated and resolved.

Open Access Statement: This is an Open Access article distributed in accordance with the Creative Commons Attribution-NonCommercial-NoDerivs 4.0 International License (CC BY-NC-ND 4.0), which permits the noncommercial replication and distribution of the article with the strict proviso that no changes or edits are made and the original work is properly cited (including links to both the formal publication through the relevant DOI and the license). See: https://creativecommons.org/licenses/by-ncnd/4.0/.

\section{References}

1. Raymond NJ, Nguyen M, Allmark S, et al. Modified Sequential Organ Failure Assessment sepsis score in an emergency department setting: Retrospective assessment of prognostic value. Emerg Med Australas 2019;31:339-46.

2. Xiao SH, Luo L, Liu XH, et al. Curative efficacy and safety of traditional Chinese medicine xuebijing injections combined with ulinastatin for treating sepsis in the Chinese population: a meta-analysis. Medicine (Baltimore) 2018;97:e10971.

3. Lu B, Wu C, Azami NLB, et al. Babao Dan improves neurocognitive function by inhibiting inflammation in clinical minimal hepatic encephalopathy. Biomed Pharmacother 2021;135:111084.

4. Lin CJ, Su YC, Lee CH, et al. Bai-hu-tang, ancient Chinese medicine formula, may provide a new complementary treatment option for sepsis. Evid Based Complement Alternat Med 2013;2013:193084.

5. Liang X, Zhou M, Ge XY, et al. Efficacy of traditional Chinese medicine on sepsis: a systematic review and metaanalysis. Int J Clin Exp Med 2015;8:20024-34.

6. Wu YP, Lauffenburger JC. Effectiveness of corticosteroids in patients with sepsis or septic shock using the new third international consensus definitions (Sepsis-3): A retrospective observational study. PLoS One 2020;15:e243149.

7. Zhang N, Liu J, Qiu Z, et al. Shenfu injection for improving cellular immunity and clinical outcome in patients with sepsis or septic shock. Am J Emerg Med 2017;35:1-6.

8. Chen XQ, Luo ZJ, Yin AZ, et al. Effect of drug enema with Ginseng Wuwei Xiaodu drink on serum inflammatory factors in patients with burn sepsis. Journal of Guangdong Medical College 2019;37:55-8.

9. Xing $\mathrm{X}, \mathrm{Zhi} \mathrm{Y}, \mathrm{Lu} \mathrm{J}$, et al. Traditional Chinese medicine bundle therapy for septic acute gastrointestinal injury: a multicenter randomized controlled trial. Complement Ther Med 2019;47:102194.

10. Zeng R, Zheng Y, Fan R, et al. Si-ni-tang (a Chinese herbal formula) for improving immunofunction in sepsis: study protocol for a pilot randomized controlled trial. Trials 2019;20:537.

11. Liu HL, Huo JY, Yan YX. Effect of Fuzheng Jiedu Decoction Combined with Shenmai Injection on the Humoral Immune Function in Patients with Sepsis. China Pharmaceuticals 2021;30:74-6.

12. Chen FQ, Xu WZ, Gao HY, et al. Clinical effect of 
Changweishu on gastrointestinal dysfunction in patients with sepsis. J Int Med Res 2020;48:300060520919579.

13. Zhang L. Clinical Effect of Rhubarb Root and Rhizome on Pyemia and the Effect on Inflammatory Factor. Guangming Journal of Chinese Medicine 2016;31:2512-3..

14. Yang G, Hu RY, Deng AJ, et al. Effects of ElectroAcupuncture at Zusanli, Guanyuan for Sepsis Patients and Its Mechanism through Immune Regulation. Chin J Integr Med 2016;22:219-24.

15. Mierzchała-Pasierb M, Lipińska-Gediga M. Sepsis diagnosis and monitoring - procalcitonin as standard, but what next? Anaesthesiol Intensive Ther 2019;51:299-305.

16. Gong P, Lu Z, Xing J, et al. Traditional Chinese medicine Xuebijing treatment is associated with decreased mortality risk of patients with moderate paraquat poisoning. PLoS One 2015;10:e0123504. Erratum in: PLoS One 2015;10:e0130508.

17. Qiu X, Zhang L, Tong Y, et al. Interleukin-6 for early

Cite this article as: Wen Y, Feng C, Chen W, Chen C, Kuang S, Liu F, Tang Q, Chen M. Effect of traditional Chinese medicine on serum inflammation and efficacy in patients with sepsis: a systematic review and meta-analysis. Ann Palliat Med 2021;10(12):12456-12466. doi: 10.21037/apm-21-3179 diagnosis of neonatal sepsis with premature rupture of the membranes: a meta-analysis. Medicine (Baltimore) 2018;97:e13146.

18. Godinjak A, Iglica A, Rama A, et al. Predictive value of SAPS II and APACHE II scoring systems for patient outcome in a medical intensive care unit. Acta Med Acad 2016;45:97-103.

19. Zhang L, Long K, Wang C, et al. Effects of Fusu mixture (Wen-Shen-Qian-Yang Method) on sepsis-induced acute respiratory distress syndrome. Medicine (Baltimore) 2020;99:e21066.

20. Wang Y, Zhang Y, Jiang R. Early traditional Chinese medicine bundle therapy for the prevention of sepsis acute gastrointestinal injury in elderly patients with severe sepsis. Sci Rep 2017;7:46015.

(English Language Editor: J. Jones) 